\title{
Studies on mean performance, correlation and path analysis coefficient of some flax genotypes under different environmental conditions.
}

\author{
EL-Shimy, G.H.: M.M.M. Hussein and Sanaa S. Hassan \\ Corresponding author: dr.sanaa59@gmail.com
}

\begin{abstract}
The present investigation was designed to evaluate sixteen flax genotypes (14 promising lines and 2 check varieties viz., Sakha 1 and Sakha 2 (dual purpose types)) for mean performance, correlation and path coefficients for straw and seed yields and some of their attributed traits under six environments (E), two seasons (2009/2010 and 2010/2011) in 3 locations (Kafr EL-Hamam Agric. Res. Station , EL-Sharkia Governorate as clay soil, Ismailia Agric. Res. Station, Ismailia Governorate as sandy soil and Tag EL-Ezz Agric. Res. Station , Duqhlia Governorate as saline soil). It also aimed to determine the superior strains which can be used as useful genetic sources in flax breeding programs.

These materials were evaluated in a randomized complete block design with three replications at the tested six environments. The analysis of variance revealed highly significant differences among genotypes (G)locations (L) and (G x L) interactions for most straw and seed yield traits, indicating a widely range of variation among genotypes, environments and these genotypes exhibited differential response to environmental conditions as average of both seasons and both locations ; S.31/3/2, followed by S.413/3/4, surpassed significantly the other tested flax genotypes in respect to straw yield traits i.e.strawyield/fed and fiber yield/fed, straw yield/plant, total and technical length /plant, fiber percentage,fiber length and fiber fineness .Sakha 1 cultivar ranked the third in all straw yield traits. However S.16 followed by S.2 had the highest seed yield traits i.e. seed yield/fed., oil yield/ fed., seed yield /plant, number of capsules/ plant,number of seeds/ capsule, 1000 seed weight and oil percentage. Sakha 2 cultivar ranked the third for seed yield traits. On the other hand, the other tested flax genotypes recorded intermediate estimates for either straw and seed yield traits. Results of simple correlation coefficient revealed that Straw yield/ fed showed positive and highly significant correlation coefficient with all Straw yield traits i.e. fiber yield /fed, straw yield /plant, total and technical length / plant, fiber percentage, fiber length and fiber fineness. In the same time seed yield/fed exhibited positive and highly significant associations with all seed yield traits i.e. oil yield /fed, seed yield/plant, number of capsules /plant, number of seed / capsules , 1000 seed weight and oil percentage. A path coefficient analysis for genotypes indicated that technical length/plant and straw yield /plant and their interaction are considered the main important sources in increasing straw yield /fed having contribution percentage of 39.21 and $9.56 \%$, respectively . In the same time, 1000 seed weight and seed yield /plant are considered the main important sources in increasing seed yield /fed, with contribution percentage of 23.29 and $11.78 \%$, respectively. Results indicated that the maximization of straw and seed yield may be obtained by selection for technical length /plant and 1000seed weight, respectively.
\end{abstract}

Key words: Flax genotypes, yield, yield components, locations, soil types, correlations, path coefficient.

\section{Introduction}

Flax (Linum usitatissmum, L.) is one of the oldest crop grown widely for its fiber, seed and dual purpose. The fibers are used in textile as well as automobile and construction industries, while flax seeds are used in human food as source of omega-3 and omega- 6 fatty acids and animal feed. Linseed oil is used in the production of paint, soap, putty and polymers (Kozlowski, 2001).

Weather conditions and other factors are important to determine yield potential of genotypes. Plant breeders noticed that different genotypes do not react in similar way to the changes of environment. It is well known that genotype $\mathrm{x}$ environment interaction $(\mathrm{G} \quad \mathrm{x}$ E) often causes inconsistent differences in genotypes response from one environment to another, which may result from two seasons; one being the differences in response of some set of genes to different and the second being the expression of various set of genes in varying environments; i.e. crossover or qualitative interaction (Baker, 1988). Choosing the promising lines or varieties and planting them in suitable location are among the most important factors affecting the productivity of flax. The ultimate goal of flax breeding program in Egypt is to improve both seed and straw yield as well as fiber and oil quality traits in the genotypes and at the same time, these genotypes has a minimum interaction with environments as stable genotypes.

Wide variability among flax genotypes regarding yield, yield components and quality was reported by several workers such as Mourad et al (1986),Satapathi et al (1987) and Mourad et al (2003).

Genotypes $\mathrm{x}$ environment interaction $(\mathrm{G} \times \mathrm{E})$ is an important consideration in breeding programs. If 
it is large, a breeder may have to test the performance of genotypes in several environments such as different locations. Similar workers studied G x E interactions interaction of flax genotypes under different locations, among of them, Abo-EL-Zahab et al (1994), Mahto (1995), Abo.Kaied et al (2008) and Abo.Kaied et al (2011).

Mean performance among flax genotypes, in respect to yield and quality, were reported by several investigators, among of them, Halla (1983), ELHariri et al (2002a,b), Mostafa and Ashmawy (2003), EL-Kady and Kineber (2004), EL-Kady and AbdelFattah (2009), EL-Refaie et al (2011)and Hussein (2012).

The correlation coefficient is one of the most important measurements for the degree of association between any two traits, thus it is applied in plant breeding programs. Correlation coefficient between yield and yield components, varying in magnitude and direction were reported in different flax yield studies by several workers, among them Kedhamath et al (1960), Badwal et al (1971), Momtaz et al (1977), Kumar and Chauhan (1979), Patial et al (1998), Abo-kaied et al (2011) and Hussein (2012).

Path coefficient analysis were performed to estimate the relative contribution of yield components of flax straw, fiber and seed yield variation by several workers, among them, Mourad et al (1986), Satapathi et al (1987), Muduli and Patmaik (1994), Aly and Awaad (1997), AL-Kaddoussi and Moawed (2001) and Hussein(2012).

Therefore, the main objectives of the present study were to 1- Evaluate sixteen flax genotypes for yield and yield components 2- To identify the effects of environments and genotypes by environment interaction, to detect the direct and indirect effects of straw and seed yield components on straw 3-Seed yields as well as the important contributing traits in flax genotypes.

\section{Materials and methods}

\section{A- Description of the studied materials:}

Six field experiments were carried out during 2009/2010 and 2010/2011 seasons at three locations namely Kafr EL-Hamam Agric. Res. Station as clay soil (L1), Ismailia Agric. Res. Station as sandy soil (L2) and Tag EL-Ezz Agric. Res. Station as saline soil (L3) to evaluate the performance of sixteen flax genotypes for yield and yield components, to estimate correlation and path coefficient analysis for straw and seed yields and some of most important contributing traits. Prior to sowing, the physical and chemical properties of soil of the three experimental sites were analyzed mechanically following the method described by Piper (1950) and chemically according to Black et al (1965). The soil characteristics in the three experimental sites were presented in table (1). The tested sixteen flax genotypes (Linum usitatissmum, L.) included Sakha 1 and Sakha 2 as check varieties (dual purpose types) in addition to 14 promising strains i.e.S1,S3,S5,S31/3/2, S421/3/6/5 and S413/ $3 / 4$ as dual purpose strains and S2419/1, S2465/1/3, S2, S4, $\mathrm{S} 16, \mathrm{~S} 402 / 3 / 1819, \mathrm{~S} 402 / 3 / 5 / 1$ and $\mathrm{S} 402 / 3 / 8 / 1$, as oil strains.

Pedigree and origin of the sixteen flax genotypes under study are presented in table (2).

Table1. Some physical and chemical properties of representative soil samples in the experimental soil before sowing (0-30 cm depth) in 2009 / 2010 and 2010/ 2011 seasons.

\begin{tabular}{|c|c|c|c|c|c|c|c|c|c|c|c|c|}
\hline \multirow{3}{*}{ 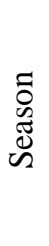 } & \multirow{3}{*}{$\begin{array}{l}\text { Variable } \\
\text { Location }\end{array}$} & \multicolumn{6}{|c|}{ Physical properties } & \multicolumn{5}{|c|}{ Chemical properties } \\
\hline & & \multirow{2}{*}{ 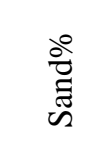 } & \multirow{2}{*}{$\frac{\text { bo }}{\stackrel{ \pm}{n}}$} & \multirow{2}{*}{$\frac{0^{0}}{\stackrel{\vec{\Xi}}{U}}$} & \multirow{2}{*}{$\sum_{0}^{\infty}$} & \multirow{2}{*}{ 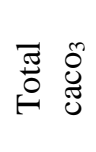 } & \multirow{2}{*}{ 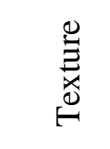 } & \multirow{2}{*}{ 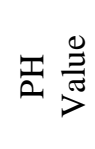 } & \multirow{2}{*}{ 讷 } & \multicolumn{3}{|c|}{ Available (ppm) } \\
\hline & & & & & & & & & & $\mathrm{N}$ & $\mathrm{P}$ & $\mathrm{K}$ \\
\hline \multirow{3}{*}{ 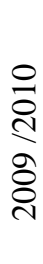 } & $\begin{array}{l}\text { KafrEL- } \\
\text { Hamam }\end{array}$ & 25.62 & 28.56 & 45.82 & 2.31 & 2.55 & Clay & 8.11 & 3.15 & 64.15 & 29.20 & 325.69 \\
\hline & Ismailia & 69.21 & 7.92 & 22.87 & 1.25 & 1.56 & Sandy & 8.42 & 1.26 & 29.55 & 31.55 & 32.76 \\
\hline & Tag EL-Ezz & 36.60 & 22.28 & 41.12 & 0.40 & 3.25 & Clay & 6.45 & 4.82 & 45.12 & 20.15 & 256.25 \\
\hline \multirow{3}{*}{ 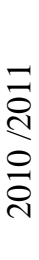 } & $\begin{array}{c}\text { Kafr EL- } \\
\text { Hamam }\end{array}$ & 22.18 & 27.75 & 49.87 & 2.84 & 2.66 & Clay & 8.25 & 4.74 & 66.88 & 31.22 & 336.25 \\
\hline & Ismailia & 63.54 & 12.96 & 23.50 & 1.55 & 1.64 & Sandy & 8.86 & 1.56 & 31.48 & 35.39 & 36.50 \\
\hline & Tag EL-Ezz & 28.15 & 24.16 & 47.69 & 0.92 & 3.42 & Clay & 6.72 & 5.35 & 46.11 & 25.12 & 264.25 \\
\hline
\end{tabular}


Table 2. Pedigree of the sixteen flax genotypes (Linum usitatissmum, L.) used under study:

\begin{tabular}{ccccc}
\hline No. & Genotypes & Pedigree & Type & Origin \\
\hline 1 & Sakha 1 (c.v.) & Bombay (USA) x I. 1485 (USA) & Dual & Local variety \\
2 & Sakha 2 (c.v.) & I.2348 (Hungar) x I. Hera(India) & Dual & Local variety \\
3 & S. 1 & I. 2467 x I. 65. & Dual & Local strain \\
4 & S. 3 & I. $2348 \times$ Hera & Dual & Local strain \\
5 & S. 5 & I. $2351 \times$ F. $268 / 0$ & Dual & Local strain \\
6 & S. $31 / 3 / 2$ & S. $402 / 21 / 19 / 3 \times$ S. $400 / 5 / 6$ & Dual & Local strain \\
7 & S. $421 / 3 / 6 / 5$ & S. $162 / 12 \times$ S. 612 & Dual & Local strain \\
8 & S. $413 / 3 / 4$ & S. $5282 / 1 \times$ S. $40 / 9$ & Dual & Local strain \\
9 & S. $2419 / 1$ & Selected from I. Humata (Hungarian) & Oil & Local strain \\
10 & S. $2465 / 1 / 3$ & Selected from I. Neelum (India) & Oil & Local strain \\
11 & S. 2 & Bombay x I. 1485 & Oil & Local strain \\
12 & S. 4 & I. 2348 x Hera & Oil & Local strain \\
13 & S. 16 & Giza $8 \times$ S.24/9/1 & Oil & Local strain \\
14 & S. $402 / 3 / 18 / 9$ & Giza $5 \times$ I. 235 (USA) & Oil & Local strain \\
15 & S. $402 / 3 / 5 / 1$ & Giza $5 \times$ I. 235 (USA) & Oil & Local strain \\
16 & S. $402 / 3 / 8 / 1$ & Giza $5 \times$ I. 235 (USA) & Oil & Local strain \\
\hline
\end{tabular}

B- Layout and experimental design:

A randomized complete block design with three replications was used at each location. Plot size was $6 \mathrm{~m}^{2}(2 \mathrm{x} 3)$ in both seasons. Seeds of the sixteen tested flax genotypes were hand drilled into rows 15 $\mathrm{cm}$ apart at sowing rate of $70 \mathrm{~kg}$ seeds / fed during the period from 15 to 18 November in both seasons. Seeds of the sixteen tested flax genotypes were obtained from Fiber Crops Research Section, Field Crops Research Institute, ARC. Recommended P and $\mathrm{K}$ fertilizers were pre sowing fully at the rate of 100 $\mathrm{kg} /$ fed calcium superphosphate $\left(\begin{array}{lll}15.5 \% & \left.\mathrm{P}_{2} \mathrm{O}_{5}\right)\end{array}\right)$ and added $24 \mathrm{~kg} /$ fed potassium sulphate $\left(48.5 \% \mathrm{~K}_{2} \mathrm{O}\right)$ for Kafr EL-Hamam and Tag EL-Ezz location (clay soil) and $150 \mathrm{~kg} / \mathrm{fed}$ calcium superphosphate $(15.5 \%$ $\left.\mathrm{P}_{2} \mathrm{O}_{5}\right)$ and $50 \mathrm{~kg} / \mathrm{fed}$ potassium sulphate $\left(48.5 \% \mathrm{~K}_{2} \mathrm{O}\right)$ for Ismailia location (sandy soil).

Recommended $\mathrm{N}$ fertilizer was applied at the rate of $45 \mathrm{~kg} / \mathrm{fed}$ in the form of ammonium nitrate $(33.5 \% \mathrm{~N})$ in two equal doses for Kafr EL-Hamam and Tag EL-Ezz location. However, $\mathrm{N}$ fertilizer was added at the rate of $75 \mathrm{~kg} \mathrm{~N} / \mathrm{fed}$ in the form of ammonium sulphate $(20.5 \% \mathrm{~N})$ in four equal doses for Ismailia location, the first dose was applied before the first irrigation, while the remainder were added at 30 day intervals for Kafr EL-Hamam and Tag EL-Ezz location and 15day intervals for Ismailia location.

Weeds were mechanically controlled. Irrigation was carried out using flooding irrigation in the three locations. The preceding crop was corn, peanut and rice in the two seasons at the three locations respectively. All other recommended agronomic practices of flax growing were followed for the region.

\section{Recorded data:}

At full maturity, ten guarded plants in each experimental unit in the three replications of the three locations were hand pulled carefully at random and left one week for complete air drying to determine yield components of flax. However, yields of straw, fiber and seed per feddan where calculated from a control area of $2 \mathrm{~m}^{2}$ of each subplot, which estimated in $\mathrm{kg} / \mathrm{m}^{2}$, and then converted to $(\mathrm{kg} / \mathrm{fed})$. The retting process was carried out in Fiber Crops Research Section, Field Crops Research Institute, ARC, to extract flax fiber for studying its quantity and quality parameters.Seed oil percentage was determined by using Soxhelt apparatus and using pure petroleum ether as a solvent according to A.O.A.C.(1995), Oil yield (kg/fed) was calculated by multiplying seed oil percentage $\mathrm{x}$ seed yield/fed.

\section{Data Calculated Included:}

\section{A-Yield and yield components:}

1-Straw yield and its related characters:total length /plant $(\mathrm{cm})$,technical stem length $(\mathrm{cm})$,straw yield/plant (g),straw yield/fed (ton),fiber yield/fed (ton),fiber percentage (\%) it was determined as follows:

$$
\text { Fiber } \%=\frac{\text { Fiber yield } / \text { fed }}{\text { Retted straw yield } / \text { fed }} x 100
$$


Fiber length $(\mathrm{cm})$ : it was measured as average of ten fiber ribbons (bundles) from each subplot, fineness (N. m): It was determined according to Radwan and Momtaz (1966) as follows:

$N . m=(N x L) / G$

Where N.m = metrical number, $\mathrm{N}=$ number of 20 fibers in $\mathrm{mm}(2000)$ and $\mathrm{G}=$ weight of fibers in $\mathrm{mg}$.

2- Seed yield and its related characters: fruiting zone length $(\mathrm{cm})$, number of capsules /plant, number of seeds/capsule, 1000 seed weight $(\mathrm{g})$,seed yield /plant (g),seed yield /fed (kg), oil percentage (\%) and oil yield /fed (kg).

\section{B-Correlation coefficient analysis study:}

The association between straw and seed yield/fed and their attributes as average of the two seasons were subjected to simple correlation coefficient according to Svab (1973)

\section{C- Path coefficient analysis study:}

Path coefficient analysis could be successfully used to identify appropriate selection criteria for improving fiber and oil yields. In this study path coefficient analysis was calculated from the combined data over the two seasons and the three locations by partitioning the simple correlation coefficient between:

- Straw yield /fed and its components i.e. fiber yield/fed., straw yield /plant, total length and technical length/plant, fiber percentage, fiber length and fiber fineness.

- Seed yield/ fed and its components i.e. oil yield/fed.seed yield /plant, number of capsules/plant, number of seeds /capsules, 1000 seed weight and oil percentage.

The Path coefficient analysis was computed by using the method mentioned by $\mathrm{Li}$ (1975). It was performed to estimate the relative contribution of yield components of flax straw and seed yield variations.

\section{Statistical analysis:}

Plot means were used for statistical analysis. Data from each of the six environments (Combination of year and locations) were analyzed. Bartlett test of homogeneity was used before combined analysis. The data obtained were subjected to the proper statistical analysis as a randomized complete block design according to Snedcor and Cochran (1982) and treatment means were compared using LSD at 5\% level of probability in both seasons, The combined analysis was performed for each character over the two seasons as described by Le Clerg et al (1966).

\section{Results and discussion}

\section{I- Mean performance:}

A- Yield and yield components:

\section{I-1- Straw yield and its related characters:}

Results of straw yield traits i.e. straw yield / fed., fiber yield/fed, straw yield/plant, total length/plant, technical length/plant, fiber\%, fiber length and fiber fineness are shown in Table (3).

The analysis of variance showed that the studied flax genotypes differed significantly in all previous straw yield traits as average of three locations and two seasons ( six environments). Data obtained from the combined analysis exhibited that S.31/3/2 ranked first in all eight straw traits estimates at same regularity which mentioned before with the mean values of 4.657 ton/fed., 0.548 ton/fed., 2.334 $\mathrm{g} /$ plant, $111.30 \mathrm{~cm}, 92.23 \mathrm{~cm}, 16.81 \% 91.04 \mathrm{~cm}$ and 236.76 N.m, respectively. The respective estimates for S. 413/3/4 which occupied the second positive in descending order were 4.17 ton/fed.,0.530 ton/fed., $2.211 \mathrm{gm} /$ plant, $109.06 \mathrm{~cm}, 89.97 \mathrm{~cm}, 16.40 \% 89.11$ $\mathrm{cm}$ and 212.83 N.m. Meanwhile, the lowest mean performance obtained by $S 402 / 3 / 8 / 1$ with the respective mean values of 1.689 ton/fed.,0.131 ton/fed., $0.762 \mathrm{gm} /$ plant, $62.35 \mathrm{~cm}, 47.88 \mathrm{~cm}, 10.26$ $\% 46.28 \mathrm{~cm}$ and 130.59 N.m., respectively. The reminder flax genotypes laid the intermediate situation between the highest two flax strains (S.31/3/2 and S. 413/3/4) and the fewest one $\mathrm{S} 402 / 3 / 8 / 1$. Moreover, the superiority ratios between S.31/3/2 and the lowest one 402/3/8/1 concerning all straw yield traits were $175.73 \%$ for straw yield /fed., $356.49 \%$ for fiber yield/ fed., $207.89 \%$ f0r straw yield / plant, $78.51 \%$ for total length / plant, $92.63 \%$ for technical length / plant, $63.84 \%$ for fiber percentage, $98.66 \%$ for fiber length $81.30 \%$ for fiber fineness. As average of the two seasons and the three locations respectively. These results are in accordance with those obtained by Abo EL-Zahab et al (1994), EL-Hariri et al (2002a), Mostafa and Ashmawy (2003), EL-Kady and Kineber (2004), Abo-Kaied et al (2008) EL-Refaie et al (2011) and Hussein (2012).

Generally the promising strain S.31/3/2 achieved the highest mean performance over all studied locations regarding straw yield characters. Therefore, it must be recommended to release on high yielding new flax variety.

\section{2- Seed yield and its related characters:}

Mean performance for seed yield / fed, oil yield /fed, seed yield/plant, number of capsules/plant, number of seeds /capsule, 1000 seed weight and oil percentage of sixteen flax genotypes average over six environments are presented in Table (4). 
Table 3. Mean squares of combined analysis over six environments ( 3 locations x 2 seasons) for straw yield and its related characters of sixteen flax genotypes

\begin{tabular}{|c|c|c|c|c|c|c|c|c|c|}
\hline SOV & d.f & $\begin{array}{l}\text { Straw yield/fed } \\
\text { (ton) }\end{array}$ & $\begin{array}{l}\text { Fiber yield/fed } \\
\text { (ton) }\end{array}$ & $\begin{array}{c}\text { Straw yield / } \\
\text { plant } \\
(\mathrm{gm}) \\
\end{array}$ & $\begin{array}{l}\text { Total length } \\
\text { /plant }(\mathrm{cm})\end{array}$ & $\begin{array}{l}\text { Technical stem } \\
\text { length }(\mathrm{cm})\end{array}$ & Fiber $\%$ & $\begin{array}{l}\text { Fiber length } \\
\quad(\mathrm{cm})\end{array}$ & $\begin{array}{c}\text { Fiber fineness } \\
\text { (N.m) }\end{array}$ \\
\hline Year (Y) & 1 & 3.87 & $0.23 * *$ & $1.52 * *$ & $695.80 * *$ & $304.37 * *$ & $39.60 * *$ & $285.11 *$ & $2046.65 * *$ \\
\hline Location (L) & 2 & 16.99 & $1.48 * *$ & $7.57 * *$ & $1367.62 * *$ & $902.85 * *$ & $389.56 * *$ & $871.86 * *$ & $6161.68 * *$ \\
\hline Lx Y & 2 & 0.03 & 0.006 & 0.007 & $4.90 * *$ & 2.69 & 0.38 & 1.63 & 332.09 \\
\hline$L \times Y \times R$ & 12 & $0.29 * *$ & 0.006 & 0.05 & $35.59 * *$ & 3.82 & 0.51 & 4.65 & 62.11 \\
\hline Genotypes (G) & 15 & $19.88 * *$ & $0.65 * *$ & $15.08 * *$ & $304.88 * *$ & $349.29 * *$ & $81.35^{* *}$ & $347.13 * *$ & $1603.28 * *$ \\
\hline $\mathrm{L} \times \mathrm{G}$ & 30 & 0.11 & $0.02 * *$ & 0.18 & $21.87 *$ & $25.47 * *$ & 0.60 & 25.12 & 120.03 \\
\hline$Y \times G$ & 15 & 0.10 & 0.004 & 0.09 & 2.77 & 1.90 & 0.02 & 2.30 & 85.88 \\
\hline$L \times Y \times G$ & 30 & 0.01 & 0.003 & 0.067 & 0.03 & 0.04 & 0.007 & 0.06 & 35.05 \\
\hline Error & 180 & 0.09 & 0.003 & 0.14 & 12.47 & 10.07 & 0.44 & 13.60 & 111.60 \\
\hline
\end{tabular}

$*, * *$ and ns refer to significant at $5 \%$ and $1 \%$ level of probability and insignificant respectively. 
Table 4. Mean squares of combined analysis over six environments (3 locations x 2 seasons) for straw yield and its related characters of sixteen flax genotypes

\begin{tabular}{|c|c|c|c|c|c|c|c|c|c|}
\hline $\mathrm{SOV}$ & d.f & $\begin{array}{c}\text { Seed yield/fed } \\
(\mathrm{kg})\end{array}$ & $\begin{array}{c}\text { Oil yield/fed } \\
(\mathrm{kg})\end{array}$ & $\begin{array}{c}\text { Seed yield / } \\
\text { plant } \\
(\mathrm{gm})\end{array}$ & $\begin{array}{l}\text { Fruiting zone } \\
\text { length }(\mathrm{cm})\end{array}$ & $\begin{array}{c}\text { Number of } \\
\text { capsules/pant }\end{array}$ & $\begin{array}{c}\text { Number of } \\
\text { seeds/capsule }\end{array}$ & $\begin{array}{c}1000 \text { Seed } \\
\text { weight }(\mathrm{gm})\end{array}$ & Oil \% \\
\hline Year (Y) & 1 & $13592.55 * *$ & $2970.40 * *$ & $0.98 * *$ & 84.98 & $42.24 * *$ & $2.76^{* *}$ & $20.83^{* *}$ & $43.27 * *$ \\
\hline Location (L) & 2 & $76251.25 * *$ & $41485.99 * *$ & $10.81 * *$ & $468.99 * *$ & $603.68 * *$ & $99.96 * *$ & $110.83^{* *}$ & $2346.35^{* *}$ \\
\hline L x Y & 2 & 499.99 & 719.43 & 0.005 & 0.71 & 0.219 & 0.02 & 0.15 & 3.97 \\
\hline$L \times Y \times R$ & 12 & 737.99 & 227.10 & 0.141 & 17.06 & 2.47 & 0.08 & 0.76 & 5.99 \\
\hline Genotypes (G) & 15 & $21131.93^{* *}$ & $4565.93 * *$ & $14.04 * *$ & 259.16 & $296.08 * *$ & $1.72 * *$ & $11.36^{* *}$ & $65.79 * *$ \\
\hline Lx G & 30 & 1817.37 & $1578.53 * *$ & 0.095 & 2.37 & $3.90 * *$ & 0.08 & 0.08 & 4.29 \\
\hline$Y \times G$ & 15 & 2263.74 & 543.57 & 0.029 & 0.58 & 2.27 & 0.11 & 0.16 & 5.81 \\
\hline$L \times Y \times G$ & 30 & 135.66 & 223.06 & 0.005 & 0.13 & 0.71 & 0.02 & 0.002 & 2.65 \\
\hline Error & 180 & 2377.04 & 526.24 & 0.082 & 23.81 & 1.96 & 0.18 & 2.48 & 3.89 \\
\hline
\end{tabular}

$*, * *$ and ns refer to significant at $5 \%$ and $1 \%$ level of probability and insignificant respectively. 
Table 3. Mean performance of Straw yield and its related characters of sixteen flax genotypes by three locations (Combined analysis of 2009/2010 and 2010/2011 seasons).

\begin{tabular}{|c|c|c|c|c|c|c|c|c|c|c|c|c|c|c|c|c|}
\hline Characters & \multicolumn{4}{|c|}{$\begin{array}{l}\text { Straw yield/fed } \\
\text { (ton) }\end{array}$} & \multicolumn{4}{|c|}{$\begin{array}{l}\text { Fiber yield/fed } \\
\text { (ton) }\end{array}$} & \multicolumn{4}{|c|}{$\begin{array}{l}\text { Straw yield / plant } \\
\text { (gm) }\end{array}$} & \multicolumn{4}{|c|}{ Total length /plant $(\mathrm{cm})$} \\
\hline $\begin{array}{l}\text { Location } \\
\text { Genotypes }\end{array}$ & L1 & $\mathrm{L} 2$ & L3 & Mean & $\mathrm{L} 1$ & L2 & L3 & Mean & L1 & $\mathrm{L} 2$ & L3 & Mean & L1 & $\mathrm{L} 2$ & $\mathrm{~L} 3$ & Mean \\
\hline Sakha 1 (c.v.) & 4.60 & 3.87 & 3.45 & 3.98 & 0.85 & 0.59 & 0.48 & 0.64 & 2.19 & 1.84 & 1.64 & 1.89 & 120.34 & 101.90 & 91.52 & 104.58 \\
\hline Sakha 2 (c.v.) & 4.13 & 3.47 & 3.09 & 3.57 & 0.75 & 0.53 & 0.42 & 0.57 & 1.89 & 1.59 & 1.42 & 1.64 & 106.93 & 90.64 & 81.46 & 93.01 \\
\hline S. 1 & 3.50 & 2.94 & 2.62 & 3.02 & 0.60 & 0.42 & 0.34 & 0.45 & 1.73 & 1.45 & 1.29 & 1.49 & 94.17 & 79.63 & 71.88 & 81.90 \\
\hline S. 3 & 3.86 & 3.24 & 3.05 & 3.39 & 0.68 & 0.48 & 0.41 & 0.53 & 1.82 & 1.53 & 1.37 & 1.57 & 99.33 & 83.45 & 74.51 & 85.76 \\
\hline S. 5 & 3.78 & 3.18 & 2.54 & 3.27 & 0.69 & 0.49 & 0.39 & 0.52 & 1.68 & 1.56 & 1.26 & 1.49 & 96.26 & 81.03 & 72.20 & 83.16 \\
\hline S. $31 / 3 / 2$ & 5.39 & 4.53 & 4.05 & 4.66 & 1.05 & 0.74 & 0.59 & 0.79 & 2.95 & 2.23 & 1.84 & 2.34 & 128.12 & 108.4 & 47.35 & 111.30 \\
\hline S. $421 / 3 / 6 / 5$ & 4.82 & 4.06 & 3.63 & 4.18 & 0.94 & 0.66 & 0.53 & 0.71 & 2.57 & 2.14 & 1.92 & 2.21 & 125.53 & 106.25 & 95.41 & 109.06 \\
\hline S. $413 / 3 / 4$ & 3.46 & 2.91 & 2.59 & 2.99 & 0.57 & 0.40 & 0.32 & 0.43 & 1.41 & 1.19 & 1.06 & 1.22 & 94.33 & 79.25 & 70.76 & 81.45 \\
\hline S. $2419 / 1$ & 3.26 & 2.60 & 2.33 & 2.73 & 0.52 & 0.42 & 0.28 & 0.41 & 1.27 & 1.06 & 0.95 & 1.09 & 90.37 & 76.72 & 69.04 & 78.71 \\
\hline S. $2465 / 1 / 3$ & 2.88 & 2.42 & 2.16 & 2.49 & 0.44 & 0.31 & 0.25 & 0.33 & 1.22 & 1.02 & 0.91 & 1.05 & 90.80 & 76.28 & 68.11 & 78.40 \\
\hline S. 2 & 2.74 & 2.31 & 2.06 & 2.37 & 0.41 & 0.29 & 0.23 & 0.31 & 1.49 & 1.03 & 0.61 & 1.04 & 90.60 & 75.32 & 66.72 & 77.55 \\
\hline S. 4 & 2.41 & 2.02 & 1.85 & 2.09 & 0.33 & 0.24 & 0.19 & 0.25 & 1.06 & 0.89 & 0.79 & 0.92 & 86.88 & 73.78 & 66.42 & 75.69 \\
\hline S. 16 & 2.28 & 1.92 & 1.72 & 1.98 & 0.31 & 0.22 & 0.18 & 0.24 & 1.04 & 0.87 & 0.78 & 0.89 & 87.09 & 73.17 & 65.32 & 75.19 \\
\hline S. $402 / 3 / 18 / 9$ & 2.18 & 1.83 & 1.65 & 1.88 & 0.29 & 0.20 & 0.16 & 0.22 & 1.03 & 0.85 & 0.66 & 0.85 & 84.45 & 71.75 & 64.60 & 73.60 \\
\hline S. $402 / 3 / 5 / 1$ & 2.08 & 1.75 & 1.56 & 1.79 & 0.26 & 0.18 & 0.15 & 0.19 & 0.97 & 0.83 & 0.73 & 0.84 & 81.31 & 69.11 & 62.24 & 70.88 \\
\hline S. $402 / 3 / 8 / 1$ & 1.95 & 1.64 & 1.47 & 1.69 & 0.23 & 0.16 & 0.13 & 0.18 & 0.88 & 0.74 & 0.66 & 0.76 & 77.21 & 65.66 & 54.16 & 62.35 \\
\hline Mean & 3.34 & 2.79 & 2.51 & 2.88 & 0.56 & 0.39 & 0.32 & 0.42 & 1.57 & 1.30 & 1.12 & 1.33 & 97.11 & 81.96 & 73.23 & 83.91 \\
\hline LSD 5\% (L) & & & & 0.08 & & & & 0.02 & & & & 0.11 & & & & 1.01 \\
\hline $\operatorname{LSD} 5 \%(\mathrm{G})$ & & & & 0.19 & & & & 0.04 & & & & 0.25 & & & & 2.32 \\
\hline LSD 5\% (L x G) & & & & 0.34 & & & & 0.06 & & & & 0.42 & & & & 4.02 \\
\hline
\end{tabular}


Table 3. Mean performance of Straw yield and its related characters of sixteen flax genotypes by three locations (Combined analysis of $2009 / 2010$ and $2010 / 2011$ seasons).

\begin{tabular}{|c|c|c|c|c|c|c|c|c|c|c|c|c|c|c|c|c|}
\hline \multirow{2}{*}{$\begin{array}{l}\text { Characters } \\
\text { Location } \\
\text { Genotypes }\end{array}$} & \multicolumn{4}{|c|}{ Technical stem length (cm) } & \multicolumn{4}{|c|}{ Fiber $\%$} & \multicolumn{4}{|c|}{ Fiber length $(\mathrm{cm})$} & \multicolumn{4}{|c|}{ Fiber fineness (N.m) } \\
\hline & L1 & L2 & L3 & Mean & L1 & L2 & L3 & Mean & L1 & L2 & L3 & Mean & L1 & L2 & L3 & Mean \\
\hline Sakha 1 (c.v.) & 99.89 & 83.92 & 74.93 & 86.24 & 18.38 & 15.44 & 13.78 & 15.86 & 97.34 & 81.77 & 73.01 & 84.04 & 226.07 & 203.92 & 182.07 & 204.02 \\
\hline Sakha 2 (c.v.) & 87.10 & 73.17 & 65.33 & 75.20 & 18.25 & 15.33 & 13.68 & 15.75 & 84.77 & 70.79 & 63.21 & 72.92 & 232.93 & 195.68 & 174.72 & 201.11 \\
\hline S. 1 & 78.21 & 65.71 & 58.67 & 65.53 & 17.17 & 14.42 & 12.88 & 14.83 & 77.21 & 64.86 & 57.92 & 66.66 & 212.43 & 178.46 & 159.34 & 183.41 \\
\hline S. 3 & 83.19 & 69.88 & 62.42 & 71.82 & 17.72 & 14.89 & 13.29 & 15.30 & 82.20 & 69.06 & 61.66 & 70.98 & 226.49 & 190.28 & 169.89 & 195.55 \\
\hline S. 5 & 81.21 & 68.22 & 60.91 & 70.12 & 18.21 & 15.30 & 13.66 & 15.72 & 79.62 & 66.89 & 59.73 & 68.75 & 219.43 & 184.35 & 164.59 & 189.46 \\
\hline S. $31 / 3 / 2$ & 106.82 & 89.74 & 80.14 & 92.23 & 19.47 & 16.35 & 14.60 & 16.81 & 105.44 & 88.58 & 79.09 & 91.04 & 274.39 & 230.55 & 205.35 & 236.76 \\
\hline S. $421 / 3 / 6 / 5$ & 104.21 & 87.55 & 78.17 & 89.97 & 18.99 & 15.96 & 14.25 & 16.40 & 103.21 & 86.71 & 77.42 & 89.11 & 246.95 & 206.07 & 185.48 & 212.83 \\
\hline S. $413 / 3 / 4$ & 72.49 & 60.90 & 54.37 & 62.59 & 16.36 & 13.74 & 12.27 & 14.12 & 73.88 & 62.07 & 55.42 & 63.79 & 199.65 & 167.73 & 149.76 & 172.38 \\
\hline S. $2419 / 1$ & 65.65 & 54.35 & 47.99 & 61.00 & 15.99 & 13.44 & 12.02 & 13.82 & 70.75 & 59.44 & 53.07 & 61.09 & 197.81 & 166.18 & 148.38 & 170.79 \\
\hline S. $2465 / 1 / 3$ & 66.48 & 55.85 & 49.87 & 57.40 & 15.21 & 12.78 & 11.65 & 13.21 & 68.60 & 57.63 & 51.45 & 59.23 & 195.93 & 160.82 & 143.59 & 166.78 \\
\hline S. 2 & 64.37 & 54.08 & 48.29 & 55.58 & 14.79 & 12.43 & 11.09 & 12.72 & 64.88 & 54.5 & 48.67 & 56.02 & 180.37 & 151.53 & 135.30 & 155.73 \\
\hline S. 4 & 65.54 & 53.46 & 46.67 & 55.22 & 13.9 & 11.68 & 10.42 & 12.00 & 62.60 & 52.59 & 46.96 & 54.05 & 174.00 & 146.18 & 130.52 & 150.23 \\
\hline S. 16 & 63.10 & 53.01 & 47.33 & 54.48 & 13.69 & 11.50 & 10.27 & 11.82 & 62.16 & 52.22 & 46.63 & 53.67 & 173.65 & 145.84 & 130.26 & 149.92 \\
\hline S. $402 / 3 / 18 / 9$ & 60.26 & 51.05 & 45.58 & 52.30 & 13.08 & 10.99 & 9.81 & 11.29 & 59.43 & 49.93 & 44.58 & 51.32 & 168.33 & 141.41 & 126.26 & 145.33 \\
\hline S. $402 / 3 / 5 / 1$ & 58.77 & 49.38 & 44.13 & 50.76 & 12.42 & 10.43 & 9.31 & 10.72 & 57.38 & 48.21 & 43.04 & 49.55 & 160.43 & 134.79 & 120.34 & 138.52 \\
\hline S. $402 / 3 / 8 / 1$ & 55.54 & 46.57 & 41.59 & 47.88 & 11.89 & 9.99 & 8.92 & 10.26 & 53.60 & 45.03 & 40.20 & 46.28 & 151.37 & 126.86 & 113.55 & 130.59 \\
\hline Mean & 75.75 & 63.36 & 56.65 & 66.27 & 15.97 & 13.42 & 11.99 & 13.79 & 75.19 & 63.14 & 56.38 & 64.90 & 202.52 & 170.66 & 152.46 & 175.21 \\
\hline LSD 5\% (L) & & & & 0.90 & & & & 0.19 & & & & 1.05 & & & & 3.01 \\
\hline $\operatorname{LSD} 5 \%(\mathrm{G})$ & & & & 2.09 & & & & 0.44 & & & & 2.42 & & & & 6.94 \\
\hline LSD 5\% (L x & & & & 3.61 & & & & 0.76 & & & & 4.20 & & & & 12.03 \\
\hline
\end{tabular}


Table 4. Mean values of seed yield and its related characters of sixteen flax genotypes by three locations (Combined analysis of 2009/2010 and 2010/2011 seasons)

\begin{tabular}{|c|c|c|c|c|c|c|c|c|c|c|c|c|}
\hline \multirow{2}{*}{$\begin{array}{l}\text { Characters } \\
\text { Location } \\
\text { Genotypes } \\
\end{array}$} & \multicolumn{4}{|c|}{$\begin{array}{c}\text { Seed yield/fed } \\
(\mathrm{kg})\end{array}$} & \multicolumn{4}{|c|}{$\begin{array}{l}\text { Oil yield/fed } \\
(\mathrm{kg})\end{array}$} & \multicolumn{4}{|c|}{$\begin{array}{c}\text { Seed yield / plant } \\
(\mathrm{gm})\end{array}$} \\
\hline & $\mathrm{L} 1$ & $\mathrm{~L} 2$ & L3 & Mean & L1 & $\mathrm{L} 2$ & L3 & Mean & $\mathrm{L} 1$ & $\mathrm{~L} 2$ & L3 & Mean \\
\hline Sakha 1 (c.v.) & 724.92 & 609.01 & 543.76 & 625.40 & 312.49 & 208.77 & 166.44 & 229.24 & 6.76 & 5.68 & 5.07 & 5.84 \\
\hline Sakha 2 (c.v.) & 861.57 & 723.80 & 646.26 & 743.88 & 373.96 & 264.41 & 210.41 & 282.93 & 8.93 & 7.51 & 6.70 & 7.71 \\
\hline S. 1 & 655.44 & 565.63 & 491.64 & 570.90 & 279.39 & 182.05 & 147.83 & 203.09 & 5.78 & 4.85 & 4.33 & 4.99 \\
\hline S. 3 & 709.13 & 595.47 & 531.92 & 612.27 & 186.17 & 201.97 & 161.00 & 216.36 & 6.58 & 5.53 & 4.93 & 5.68 \\
\hline S. 5 & 700.07 & 588.13 & 525.12 & 604.44 & 218.42 & 195.28 & 158.34 & 211.68 & 6.23 & 5.23 & 4.67 & 5.38 \\
\hline S.31/3/2 & 649.65 & 545.77 & 487.18 & 560.87 & 258.23 & 182.25 & 145.25 & 195.24 & 5.56 & 4.67 & 4.17 & 4.80 \\
\hline S. $421 / 3 / 6 / 5$ & 638.52 & 536.42 & 478.95 & 551.30 & 249.86 & 196.73 & 140.58 & 195.72 & 5.30 & 4.47 & 3.99 & 4.60 \\
\hline S. $413 / 3 / 4$ & 630.42 & 529.61 & 472.88 & 544.30 & 243.62 & 171.95 & 137.07 & 184.21 & 4.72 & 3.97 & 3.54 & 4.08 \\
\hline S. $2419 / 1$ & 774.39 & 650.73 & 580.87 & 668.66 & 321.29 & 226.80 & 180.77 & 242.95 & 7.44 & 6.25 & 5.58 & 6.42 \\
\hline S. $2465 / 1 / 3$ & 739.43 & 621.13 & 554.64 & 638.40 & 304.42 & 222.18 & 171.28 & 232.62 & 7.21 & 6.06 & 5.41 & 6.23 \\
\hline S. 2 & 916.60 & 770.04 & 687.54 & 791.39 & 405.52 & 286.19 & 228.15 & 306.62 & 10.76 & 9.04 & 8.07 & 9.29 \\
\hline S. 4 & 805.47 & 676.67 & 604.18 & 695.44 & 337.19 & 237.98 & 189.72 & 254.96 & 8.84 & 7.43 & 6.63 & 7.63 \\
\hline S. 16 & 927.21 & 778.95 & 695.50 & 800.55 & 407.03 & 287.27 & 229.02 & 307.77 & 12.12 & 10.29 & 9.19 & 10.53 \\
\hline S. $402 / 3 / 18 / 9$ & 608.62 & 511.30 & 456.53 & 525.48 & 249.73 & 163.14 & 130.11 & 180.99 & 3.62 & 3.04 & 2.71 & 3.12 \\
\hline S. $402 / 3 / 5 / 1$ & 564.13 & 473.92 & 448.15 & 495.40 & 212.16 & 149.73 & 126.34 & 162.74 & 3.42 & 2.88 & 2.57 & 2.96 \\
\hline S. $402 / 3 / 8 / 1$ & 459.68 & 386.18 & 344.18 & 396.89 & 158.20 & 118.71 & 94.64 & 123.85 & 3.15 & 2.65 & 2.26 & 2.72 \\
\hline Mean & 710.33 & 597.69 & 534.37 & 614.13 & 292.54 & 205.96 & 163.66 & 220.69 & 6.65 & 5.60 & 4.99 & 5.75 \\
\hline LSD $5 \%(\mathrm{~L})$ & & & & 13.88 & & & & 6.53 & & & & 0.82 \\
\hline LSD 5\% (G) & & & & 32.05 & & & & 15.08 & & & & 1.88 \\
\hline LSD 5\% (L x G) & & & & 55.51 & & & & 26.12 & & & & 3.26 \\
\hline
\end{tabular}


Table 4. Mean values of seed yield and its related characters of sixteen flax genotypes by three locations (Combined analysis of 2009/2010 and 2010/2011 seasons) .

\begin{tabular}{|c|c|c|c|c|c|c|c|c|c|c|c|c|c|c|c|c|}
\hline \multirow{2}{*}{$\begin{array}{l}\text { Characters } \\
\text { Location } \\
\text { Genotypes }\end{array}$} & \multicolumn{4}{|c|}{ Number of capsules/pant } & \multicolumn{4}{|c|}{ Number of seeds/capsule } & \multicolumn{4}{|c|}{1000 Seed weight $(\mathrm{gm})$} & \multicolumn{4}{|c|}{ Oil \% } \\
\hline & L1 & $\mathrm{L} 2$ & L3 & Mean & L1 & $\mathrm{L} 2$ & L3 & Mean & $\mathrm{L} 1$ & $\mathrm{~L} 2$ & L3 & Mean & L1 & $\mathrm{L} 2$ & L3 & Mean \\
\hline Sakha 1 (c.v.) & 19.69 & 16.34 & 14.77 & 17.00 & 8.34 & 6.95 & 6.26 & 7.18 & 9.03 & 7.59 & 6.78 & 7.80 & 36.14 & 35.73 & 30.86 & 34.24 \\
\hline Sakha 2 (c.v.) & 20.33 & 17.08 & 15.27 & 17.56 & 9.92 & 7.07 & 6.32 & 7.27 & 9.12 & 7.67 & 6.84 & 7.88 & 41.48 & 34.85 & 31.31 & 35.81 \\
\hline S. 1 & 18.51 & 15.55 & 13.60 & 15.89 & 7.95 & 6.71 & 5.96 & 6.87 & 8.09 & 6.80 & 6.07 & 6.99 & 40.10 & 33.69 & 30.08 & 34.62 \\
\hline S. 3 & 19.13 & 16.07 & 14.63 & 16.61 & 8.19 & 6.88 & 6.14 & 7.07 & 8.63 & 7.25 & 6.47 & 7.45 & 40.35 & 33.90 & 30.26 & 34.84 \\
\hline S. 5 & 19.19 & 16.12 & 14.40 & 16.57 & 8.11 & 6.81 & 6.08 & 7.00 & 8.28 & 6.96 & 6.21 & 7.15 & 40.16 & 33.74 & 30.12 & 34.67 \\
\hline S.31/3/2 & 17.98 & 15.11 & 13.49 & 15.52 & 7.87 & 6.61 & 5.90 & 6.79 & 7.87 & 6.61 & 5.91 & 6.80 & 39.72 & 33.37 & 29.79 & 34.69 \\
\hline S. $421 / 3 / 6 / 5$ & 17.40 & 14.62 & 13.05 & 15.02 & 7.73 & 6.49 & 5.80 & 6.67 & 7.69 & 6.46 & 5.77 & 6.64 & 39.15 & 32.89 & 29.37 & 33.80 \\
\hline S. $413 / 3 / 4$ & 17.17 & 14.43 & 13.38 & 14.99 & 7.61 & 6.35 & 5.71 & 6.57 & 7.65 & 6.43 & 5.74 & 6.61 & 36.62 & 32.45 & 28.97 & 33.34 \\
\hline S. $2419 / 1$ & 27.05 & 22.73 & 21.37 & 23.72 & 9.46 & 7.95 & 7.16 & 8.19 & 9.61 & 8.07 & 7.21 & 8.30 & 43.91 & 36.89 & 32.93 & 37.91 \\
\hline S. $2465 / 1 / 3$ & 19.69 & 16.34 & 14.77 & 17.00 & 8.34 & 6.95 & 6.26 & 7.18 & 9.03 & 7.59 & 6.78 & 7.80 & 36.14 & 35.73 & 30.86 & 34.24 \\
\hline S. 2 & 23.11 & 19.41 & 17.47 & 19.99 & 8.97 & 7.54 & 6.73 & 7.74 & 9.25 & 7.77 & 6.94 & 7.99 & 43.39 & 36.56 & 32.55 & 37.50 \\
\hline S. 4 & 21.01 & 17.57 & 16.21 & 18.33 & 8.69 & 7.30 & 6.52 & 7.50 & 9.22 & 7.75 & 6.92 & 7.96 & 41.85 & 35.16 & 31.39 & 36.13 \\
\hline S. 16 & 33.96 & 28.50 & 23.62 & 28.68 & 9.65 & 8.11 & 7.24 & 8.33 & 10.18 & 8.56 & 7.64 & 8.79 & 44.21 & 37.14 & 33.16 & 38.17 \\
\hline S. $402 / 3 / 18 / 9$ & 16.42 & 13.79 & 12.32 & 14.18 & 7.18 & 6.03 & 5.44 & 6.22 & 7.55 & 6.34 & 5.66 & 6.52 & 37.96 & 31.89 & 28.47 & 32.77 \\
\hline S. $402 / 3 / 5 / 1$ & 15.55 & 12.95 & 11.68 & 13.39 & 6.59 & 5.64 & 4.94 & 5.69 & 7.38 & 6.20 & 5.54 & 6.38 & 37.59 & 31.58 & 28.20 & 32.46 \\
\hline S. $402 / 3 / 8 / 1$ & 13.98 & 11.74 & 10.98 & 12.24 & 6.42 & 5.29 & 4.82 & 5.54 & 6.87 & 5.78 & 5.15 & 5.44 & 36.60 & 30.75 & 27.45 & 31.60 \\
\hline Mean & 20.00 & 16.80 & 15.06 & 17.29 & 8.09 & 6.79 & 6.08 & 6.99 & 8.46 & 7.10 & 6.34 & 7.30 & 40.12 & 34.05 & 30.33 & 34.84 \\
\hline LSD 5\% (L) & & & & 0.40 & & & & & & & & 0.12 & & & & 0.56 \\
\hline $\operatorname{LSD} 5 \%(\mathrm{G})$ & & & & 0.92 & & & & & & & & 0.27 & & & & 1.30 \\
\hline LSD 5\% (L x G) & & & & 1.59 & & & & & & & & 0.47 & & & & 2.25 \\
\hline
\end{tabular}


The analysis of variance indicated that the studied genotypes differed significantly in all seed yield traits under study. The combined analysis illustrated that the flax strain S.16 promote maximum estimates for all the seven seed yield characters previously mentioned followed by S.2 but the lowest mean values were obtained by $\mathrm{S} .402 / 3 / 8 / 1$. Seed yield/fed. ranged from 396.89 to $800.55 \mathrm{~kg}$, oil yield/fed. ranged from 123.85 to 307.77 , seed yield/plant/g ranged from 2.72 to 10.53 , number of capsules/plant ranged from 12.24 to 28.68 , number of seeds/capsule ranged from 5.44 to 8.33 , and oil percentage ranged from 31.60 to 38.17 . The remain thirteen flax genotypes laid intermediate position between either S.16 or S.2 and the lowest one S.402/3/8/1. The superiority ratios between the mean performance of S.16 and the minimum estimates recorded by S.402/ $3 / 8 / 1$ were $101.71 \%, 148.5 \%, 287.13 \%, 134.31 \%$, $50.36 \%, 61.58 \%$, in addition to $20.79 \%$ for all seed yield traits which mentioed before in the same arrangement, as average for the two seasons and the three locations, respectively. Many workers reported that the flax genotypes were differed in seed yield traits such as, EL-Hariri et al (2002b), EL-Kady and Abdel- Fattah (2009) Mostafa and Ashmawy (2003), Mouradet al (2003), EL-Kady and Kineber (2004), and Hussein (2012). Abo-Kaied et al (2011), ELRefaie et al (2011) and Hussein (2012).

It must be mentioned that L1( Kafr EL-Hamam location) achieved the maximum mean performance in all straw and seed yield characters followed by L2 (Ismailia location) and finally the lowest mean values L3 (Tag EL-Ezz location) which consider as relatively salinity land in comparison with the other two locations.

Generally the flax strain S.16 promoted the highest seed yield and its components over all the three locations and both seasons. Thereby, it must be selected this flax strain to become a new variety.

\section{II- Correlation and path coefficient study: a- Correlation coefficient study:}

Results of simple correlation coefficient between straw and seed yield/fed and some of their associated characters for sixteen flax genotypes on based data of six environments (3Locations x 2Seasons) are shown in Tables (5 and 6).

Data presented in table (5) revealed that straw yield/fed was positive and highly significant correlated with each fiber yield/fed, straw yield/plant, total length/plant, technical length/plant, fiber\%, fiber length and fiber fineness. Also, fiber yield/fed recorded positive and highly significant association with straw yield/plant, total and technical length/plant, fiber\% and fiber fineness, likewise, straw yield/plant was strongly correlated with each of total and technical length/plant, fiber $\%$, fiber length and fiber fineness. Moreover total length/plant had positive and highly significant correlation with technical length, fiber\%, fiber length and fiber fineness. Meanwhile, technical length/plant indicated positive and highly significant correlation with fiber\%, fiber length and fiber fineness. On the other hand, fiber percentage recorded positive and highly significant association with each of fiber length and fiber fineness. Finally, fiber length had positive and highly significant association with fiber fineness. These finding are in accordance with those reported by Mourad et al (1986), Satapathi et al (1987), Agrawal et al (1994), Muludi and Patnaik (1994), Aly and Awaad (1997), EL-Shimy et al (1998), Mostfa et al (1998), AL-Kaddoussi and Moawed (2001) and Hussein(2012).

Data of simple correlation coefficients among seed yield /fed and their components in sixteen flax genotypes on based data of six environments are illustrated in Table (6). Results showed that seed yield /fed. exhibited positive and highly significant association with each of oil yield/fed, seed yield /plant, number of capsules /plant, number of seeds/capsules, 1000seed weight and oil\%.

Similarly, oil yield/fed recorded positive and highly significant correlation with seed yield/plant, number of capsules/plant, number of seeds/capsules, 1000 seed weight and oil \%. Also seed yield/plant exhibited positive and highly significant association with number of capsules /plant, number of seeds /capsules and 1000seed weight, but positive and significant associated with oil\%. Moreover, number of capsules /plant had positive and significant correlation with each of number of seeds /capsules and 1000seed weight, but positive and only significant correlation with oil\%. Also, the relationship between number of seeds /capsules and 1000seed was positive and highly significant. In addition to 1000 seed weight exhibited positive and highly significant association with oil\%. These results are in agreement with those obtained by Momtaz et al (1977), Agrawal et al (1994), Aly and Awaad (1997), EL-Shimy et al (1998), Mostfa et al (1998), AL-Kaddoussi and Moawed (2001) and Hussein(2012).

In general these results indicated that number of capsules /plant, number of seeds /capsule and 1000seed weight are the main components to improve seed yield/plant. Those results indicate the possibility of using both total and technical length/plant as a selection critria to improving straw weight/plant. Similar results were reported by Agrawal et al (1994), EL-Shamy et al (1998) and Mostfa et al (1998). 
Table 5. Simple correlation coefficient between straw yield and its related characters for sixteen flax genotypes

(Combined analysis over two seasons and three locations).

\begin{tabular}{|c|c|c|c|c|c|c|c|c|c|}
\hline & Variables & - & 1 & 2 & 3 & 4 & 5 & 6 & 7 \\
\hline $\mathrm{Y}$ & Straw yield/fed (ton) & - & $0.974 * *$ & $0.888^{* *}$ & $0.866 * *$ & $0.928 * *$ & $0.885 * *$ & $0.931 * *$ & $0.875^{* *}$ \\
\hline 1 & Fiber yield/fed(ton) & & - & $0.886^{* *}$ & $0.898 * *$ & $0.950 * *$ & $0.934 * *$ & $0.951 * *$ & $0.912^{* *}$ \\
\hline 2 & $\begin{array}{l}\text { Straw yield / } \\
\text { plant }(\mathrm{gm})\end{array}$ & & & - & $0.807 * *$ & $0.886 * *$ & $0.823 * *$ & $0.887 * *$ & $0.832 * *$ \\
\hline 3 & $\begin{array}{l}\text { Total length } \\
\text { /plant }(\mathrm{cm})\end{array}$ & & & & - & $0.937 * *$ & $0.893 * *$ & $0.932 * *$ & $0.903 * *$ \\
\hline 4 & $\begin{array}{l}\text { Technical } \\
\text { stemlength }(\mathrm{cm})\end{array}$ & & & & & - & $0.918 * *$ & $0.972 * *$ & $0.925 * *$ \\
\hline 5 & Fiber $\%$ & & & & & & - & $0.915 * *$ & $0.928 * *$ \\
\hline 6 & Fiber length $(\mathrm{cm})$ & & & & & & & - & $0.921 * *$ \\
\hline 7 & Fiber fineness (N.m) & & & & & & & & - \\
\hline
\end{tabular}

Table 6 .Simple correlation coefficient between seed yield and its related characters for sixteen flax genotypes (Combined analysis over two seasons and three locations)

\begin{tabular}{|c|c|c|c|c|c|c|c|c|}
\hline & Variables & - & 1 & 2 & 3 & 4 & 5 & 6 \\
\hline $\mathrm{Y}$ & Seed yield/fed $(\mathrm{kg})$ & & $0.952 * *$ & $0.837 * *$ & $0.862 * *$ & $0.837 * *$ & $0.859 * *$ & $0.754 * *$ \\
\hline 1 & Oil yield/fed(kg) & & - & $0.781 * *$ & $0.818 * *$ & $0.894 * *$ & $0.889 * *$ & $0.847 * *$ \\
\hline 2 & Seed yield / plant (gm) & & & - & $0.883 * *$ & $0.787 * *$ & $0.750 * *$ & $0.600 *$ \\
\hline 3 & Number of capsules/pant & & & & - & $0.811 * *$ & $0.797 * *$ & $0.669 * *$ \\
\hline 4 & Number of seeds/capsule & & & & & - & $0.864 * *$ & $0.834 * *$ \\
\hline 5 & 1000 Seed weight $(\mathrm{gm})$ & & & & & & - & $0.804 * *$ \\
\hline 6 & Oil \% & & & & & & & - \\
\hline
\end{tabular}

\section{B- Path coefficient study:}

The path coefficient procedure evaluates the main components effects and makes it possible to the nonstudied factors. By this method the components of variation due to each of these factors is available to the plant breeder. The relative contribution of various straw and seed yield components related to straw and seed yield/fed from the combined data of six environments (3 locations x 2 seasons) for sixteen flax genotypes are presented in tables (7, 8, 9 and 10).

\section{1- Path coefficient analysis related to straw yield and its components:}

The results of partitioning simple correlation coefficients among straw yield and its components for sixteen flax genotypes under six environmental conditions are shown in table (7). The highest direct effect was recorded for technical length (0.626). Meanwhile the highest indicated effect was noticed for total length through indirect effect via technical length/plant (0.586) and for straw yield/plant through indirect effect via technical length/plant which valued (0.554).These results indicated that the increasing alleles played a great role in increasing these characters through straw yield. The other indirect effects were weak, revealing that the decreasing alleles played a great role for these interactions to decrease straw yield.( Mahto et al 1995)

Table 7. Partitioning of simple correlation coefficient among straw yield and its components (combined data).

\begin{tabular}{lc}
\hline Source & Components \\
\hline Straw yield / plant & $\mathrm{P}_{1}=0.309$ \\
Direct effect & $\mathrm{P}_{2} \mathrm{r}_{12}=0.024$ \\
Indirect effect via total length/plant & $\mathrm{P}_{3} \mathrm{r}_{13}=0.554$ \\
Indirect effect via technical stem length & $=0.888$ \\
Total (ry $)$ & $\mathrm{P}_{2}=0.029$ \\
total length/plant & $\mathrm{P}_{2} \mathrm{r}_{12}=0.249$ \\
Direct effect & $\mathrm{P}_{3} \mathrm{r}_{23}=0.586$ \\
Indirect effect via Straw yield / plant(gm) & $=0.886$ \\
Indirect effect via technical stem length & \\
Total (ry $)$ & $\mathrm{P}_{3}=0.626$ \\
technical stem length & $\mathrm{P}_{2} \mathrm{r}_{13}=0.274$ \\
Direct effect & $\mathrm{P}_{3} \mathrm{r}_{23}=0.027$ \\
Indirect effect via Straw yield / plant $(\mathrm{gm})$ & $=0.928$ \\
Indirect effect via total length/plant &
\end{tabular}


Direct and joint effects of straw yield components i.e. straw yield/plant, total length and technical length/plant as well as their interactions recorded as percentage of straw yield/fed variation for sixteen flax genotypes under six environments are given in Table (8).

Results revealed that technical length/plant and straw yield/plant as well as their interaction are considered the main sources of straw yield /fed variation, having contribution percentage of 39.20, 9.56 and $34.30 \%$ respectively. Also $\mathrm{R}^{2}$ reached $88.14 \%$ of the total straw yield variation. However, the residual effect of the other straw yield components was $11.85 \%$. These results are harmony with those reported by Mourad et al (1986), Satapathi et al (1987), Aly and Awaad (1997), ALKaddousi and Moawed(2001) and Hussein (2012).

Table 8. Direct and joint effects of straw yield components presented as percentage of straw yield /plant variation of flax genotypes (combined data).

\begin{tabular}{llll}
\hline Source of variance & Components & C.D & $\%$ \\
Straw yield / plant $(\mathrm{gm})$ & $\mathrm{P}_{1}{ }^{2}$ & 0.0956 & 9.561 \\
\hline total length/plant & $\mathrm{P}_{2}{ }^{2}$ & 0.0008 & 0.088 \\
technical stem length & $\mathrm{P}_{3}{ }^{2}$ & 0.3290 & 39.206 \\
Straw yield / plant(gm) x total length/plant & $2 \mathrm{P}_{1} \mathrm{P}_{2} \mathrm{r}_{12}$ & 0.0148 & 1.485 \\
Straw yield / plant(gm) x technical stem length & $2 \mathrm{P}_{1} \mathrm{P}_{3} \mathrm{r}_{13}$ & 0.3430 & 34.308 \\
total length/plant x technical stem length & $2 \mathrm{P}_{2} \mathrm{P}_{3} \mathrm{r}_{23}$ & 0.0349 & 3.492 \\
$\mathrm{R}^{2}$ & & 0.8814 & 88.142 \\
$\mathrm{R}^{2} \mathrm{E}$ & & 0.1185 & 11.857 \\
\hline Total & & 1.0000 & 100.000 \\
\hline
\end{tabular}

2- Path coefficient analysis related to seed yield and its components:

The results of partitioning simple correlation coefficients among seed yield and its components of the sixteen flax genotypes under six environments are presented in Table (9). The highest direct effect was obtained for 1000seed weight followed by seed yield /plant with mean values of 0.482 and 0.343 respectively. Meanwhile the lowest values of direct effect was recorded for number of capsules/plant with mean value of 0.149 .The highest indirect effect was noticed for number of capsules/plant was through indirect effect via 1000 seed weight $(0.384)$ and for seed yield/plant indirect effect via 1000 seed weight (0.361).

Table9. Partitioning of simple correlation coefficient among seed yield and its components (combined data).

\begin{tabular}{ll}
\hline Source & Components \\
\hline Seed yield / plant & \\
\hline Direct effect & $\mathrm{P}_{1}=0.343$ \\
Indirect effect via Number of capsules/pant & $\mathrm{P}_{2} \mathrm{r}_{12}=0.131$ \\
Indirect effect via 1000 Seed weight(gm) & $\mathrm{P}_{3} \mathrm{r}_{13}=0.361$ \\
Total (ry) & $=0.837$ \\
Number of capsules/plant & \\
Direct effect & $\mathrm{P}_{2}=0.149$ \\
Indirect effect via Seed yield / plant(gm) & $\mathrm{P}_{2} \mathrm{r}_{12}=0.303$ \\
Indirect effect via 1000 Seed weight(gm) & $\mathrm{P}_{3} \mathrm{r}_{23}=0.384$ \\
Total (ry $)$ & $=0.837$ \\
1000 Seed weight & \\
Direct effect & $\mathrm{P}_{3}=0.482$ \\
Indirect effect via Seed yield / plant(gm) & $\mathrm{P}_{2} \mathrm{r}_{13}=0.257$ \\
Indirect effect via Number of capsules/pant & $\mathrm{P}_{3} \mathrm{r}_{23}=0.119$ \\
Total (ry3) & $=0.859$ \\
\hline
\end{tabular}

Results in Table (10) indicated that 1000 seed weight and seed yield/plant as well as their interaction are considered the main sources of seed yield /fed variation having contribution percentage of 23.28, 11.77 and $24.84 \%$ respectively. Also $\mathrm{R}^{2}$ reached $82.67 \%$ of the total seed yield variation. However the residual effect of seed yield components included in the present study was $17.32 \%$. These results are in accordance with those obtained by Mourad et al (1986), Muduli and Patnaik (1994), Aly and Awaad (1997), AL-Kaddousi and Moawed (2001) and Hussein (2012).

In general, these results indicated that technical length/plant is the main component of straw yield/plant. In the same time 1000 seed weight is the main component of seed yield/plant. These results indicate the possibility of using technical length /plant and 1000 seed weight as selection indices for 
improving straw, fiber, seed and in turn oil yield, respectively. These finding are in accordance with those reported by Satapathi et al (1987), Aly and
Awaad (1997), AL-Kaddousi and Moawed (2001) and Hussein (2012).

Table 10. Direct and joint effects of seed yield components presented as percentage of seed yield /plant variation of flax genotypes (combined data).

\begin{tabular}{llll}
\hline Source of variance & Components & C.D & \% \\
\hline Seed yield / plant (gm) & $\mathrm{P}_{1}{ }^{2}$ & 0.117 & 11.778 \\
Number of capsules/pant & $\mathrm{P}_{2}{ }^{2}$ & 0.022 & 2.230 \\
1000 Seed weight(gm) & $\mathrm{P}_{3}{ }^{2}$ & 0.232 & 23.287 \\
Seed yield / plant (gm)x Number of capsules/pant & $2 \mathrm{P}_{1} \mathrm{P}_{2} \mathrm{r}_{12}$ & 0.090 & 9.051 \\
Seed yield / plant (gm)x 1000 Seed weight(gm) & $2 \mathrm{P}_{1} \mathrm{P}_{3} \mathrm{r}_{13}$ & 0.248 & 24.842 \\
Number of capsules/pantx 1000 Seed weight(gm) & $2 \mathrm{P}_{2} \mathrm{P}_{3} \mathrm{r}_{23}$ & 0.114 & 11.487 \\
$\mathrm{R}^{2}$ & & 0.826 & 82.678 \\
$\mathrm{R}^{2} \mathrm{E}$ & & 0.173 & 17.322 \\
\hline Total & & 1.000 & 100.00 \\
\hline
\end{tabular}

\section{Conclusion}

From the previous results it could be concluded that the new promising strains S.31/3/2 and S.16 which released by Fiber Crops Research Section in Giza exceeded the other tested strains as well as the local commercial varieties Sakha 1 and Sakha 2 in straw and seed yields and their components respectively, that these two new strains were stable under three different environmental conditions. So it may be recommended to using the two new strains in breeding program as a new varieties.

Furthermore, improving and maximizing the productivity and quality for these two strains where as, plant breeder could be focalize his attention on technical length/plant and either of seed index 1000 seed weight or number of capsules/plant to improve and maximize straw and seed yields/plant, in turn, the final straw and seed yields per unit area of land.

\section{References}

Abo- EL-Zahab, A.A.; N.K.M. Mourad and H.M.H. Abo-kaied (1994). Genotypes - environment inter action and evaluation of flax genotypes. Straw yield.Proc. $6^{\text {th }}$ Con. Agron. Al-Azhar Univ., Cairo, Egypt, VOL. 1:129-153.

Abo-kaied, H.M.H.; M.A. Abd EL-Dayem, M.D.H. Dewedar (2007).Simulataneous selection for high yielding and stability of some economic flax characters. J. Agric. Sci., Mansura Univ., 32(25):3289-3301.

Abo-kaied, H.M.H.; T.A. Abou Zaied and Afaf, E.A. Zahana (2008). Evaluation of some flax genotypes for yield and yield components under different conditions. Egypt. J. Agric. Res., 86(2):597-610.

Abo-kaied, H.M.H.; Amany , M.M. EL-Refaie and E.I. EL-Deeb (2011). Evaluation of mean performance for some promising flax lines under different environmental conditions. Egypt. J.Appl. Sci., 26(9):587-600.
Agrawal, K.K.; J.P. Tiwari and K.K. Jain (1994). Correlation and regression analysis in linseed (Linum usitatissmum, L.). Advance in Plant Science, 7:2.

AL-Kaddussi, A.R. and E.A. Moawed (2001). Yield analysis of seed and straw yield components under three row spacing of some genotypes of flax (Linum usitatissmum, L.). Egypt. J. Appl.Sci., 16(21):426-441.

Aly,R.M. and H.A. Awaad (1997). Yielding ability and yield analysis of some flax genotypes grown under different sowing dates in sandy soil. Zagazig. J. Agric. Res. 24(2):199-211.

A.O.A.C.(1995). Official Methods of Analysis.16 ${ }^{\text {th }}$ ed. Association of Official Analysis Chemist. Washington, D.C., USA.

Bachireddy, V.R.; R. Payne; Jr.K.L. Chin and M.S. Kang (1992).Conventional selection versus methods that use genotype $\mathrm{x}$ environment interaction in sweet corn trials.Hortscience 27:436-438

Badwal, S.S.; K.S.Gill and H. Singh (1971). Correlation and regression studies in linseed (Linum usitatissmum, L.). Indian J. Agric. Sci., 41(5):475-478.

Baker, R.J. (1988). Tests for crossover genotypeenvironmental interaction. Canadian J. Plant Sci., 68:405-410

Black, C.A.; D.D. Evan; L.E. Ensmingr; L. White and E.E. Clark (1995).Methods of soil analysis (Combined and microbiology properties, part 2). Amer. Soc. Of Agron., USA.

EL-Hariri, D.M.; M.S. Hassanein and Amna H.H.EL-Sweify (2002a).Evaluation of some flax genotypes. 1-Seed yield, yield components and oil percentage. Annals of Agric. Sci., Moshtohor, 40 (1):13-25.

EL-Hariri, D.M.; Amna H.H.EL-Sweify and M.S. Hassanein (2002b).Evaluation of some flax genotypes 2-Straw yield, yield components and technological characters. Annals of Agric. Sci., Moshtohor, 40 (1):1-12. 
El- Kady, E.A. and A.A.E. Abd EL-Fatah (2009).Comparison of yield, its components, physical properties and chemical compositions of twelve flax genotypes. J. Agric. Res., Kafr ELSheikh Univ., 35(1):69-85.

El- Kady, E.A. and M.E.A.Kineber (2004). A comparative study on some flax genotypes $\mathrm{J}$. Agric. Res., Tanta Univ., 30(3):587-600.

EL-Refaie, Amany M.M.; E.I. EL-Deeb and H.M.H. Abo-Kaied (2011).Comparative study for yield and yield components of some flax lines with the two commercial varieties Sakha 1 and Sakha2. J. Plant Production, Mansoura Univ., 2(12):16331644.

EL-Shimy, G.M.; S.Z. Zedan and S.H.A. Mostafa (1998).Evaluation and interrelation ship studies in some flax genotypes. Fayoum J. Agric. Res. Dev., 12(1):39-51.

Hella, A.M. (1983). Analytical studies on flax type under different environmental conditions. Ph.D. thesis, Fac. Agric. Cairo, Univ.

Hussein, M.M.M. (2012). Evaluation of productivity and quality for some local and introduced flax (Linum usitatissmum, L.). varieties under different pulling dates in sandy soil. Minufiya J. Agric. Res., Vol. 37 No. 5(1):1171-1190.

Kang, M.S.; D.P. Gorman and H.N. Plam (1991).Application of a stability statistic to international maize yield traits.Theor. Appl. Gene. 81:162-165.

Kedharnath, S.; A.B. Joshi and M.G.B.R. Batcha (1960). Correlation studies in flax (Linum usitatissmum, L.). Effect of morphological grouping of types on correlation coefficient relating to yield and some of the components of yield.Indian J. Gent. And Pl. Breed., 20:58-68.

Kozlowski, R. (2001). Future trends in the production processing and application on nature fibers. Proc. $2^{\text {nd }}$ Global Work shops, Bact plants in the new Millennium, Borovest, Bulgaria.

Kumar, S. and B.P.S. Chauhan (1979).Association analysis in the segregation population of linseed.Indian J. Genet.And Pl. Breed. 39:506510.

LeClerg, E.L.; W.H. Leonard and A.G. Clark (1966).Field Plot Technique. $2^{\text {nd }}$ ed,Burgess publishing Co., Minneapolis, Minnesota, USA, PP.215-224.

Li, C.C. (1975). Path analysis primer.The Boxwool Press Pacific Grove, California, USA.
Mahto, J.L. (1995). Genotype x environment interaction stability and genetic diversity study in linseed for yield and yield attributes under dry land situation. Madras Agric. J., 82:601-605. (C.F. Pl. Breed. Abs., 66:1309.

Momtaz, A.; A.K.A. Salim and G.H. EL-Shimy (1977). Correlation studies on some flax crosses and their reciprocal in Egypt. Association studies between flax seeds yield and some other characters. Agri. Res. Rev., 55:45-55.

Mostafa, S.H.A. and F. Ashmawy (2003). Performance and yield stability of some flax genotypes. Annals. Agric. Sci., Ain Shams Univ., Cairo, 43(2):403-417.

Mostafa, S.H.A.; S.Z. Zedan and M.E.A. Kinebar (1998). Association studies between quantitative traits in some flax genotypes. Egypt. J. Appl. Sci. 13 (7):93-108.

Mourad, N.K.M.; A.M. Hella and A.I. Sahsah (1986).Variability, correlation and path coefficient analysis of yield and its components in flax (Linum usitatissmum, L.). J. Agric. Res., Tanta Univ., 13(1):38-51.

Mourad, N.K.M.; S.H.A. Mostafa and Afaf E. Zahana (2003).Yield, yield components, quality and variability assessment of some flax genotypes. Egypt. J. P1. Breed., 7(2):129-143.

Muludi, K.C. and M.C. Patnaik (1994).Charcter association and path coefficient analysis in linseed (Linum usitatissmum, L.). Orissa J. Agric., 7:5-11.

Patial, V.D.; G.Makne. and V.P. Chaudhari (1980). Association analysis in linseed.Dep. Agric. Bot. Maharashtra, India CC.F.Field Crop Abst. 35(6):6853.

Piper, C.S. (1950).Soil and plant analysis. Inter Science Publishers, Inc. New York, USA.

Radwan, S.R. and A. Momtaz (1966). The technological properties of flax fiber and method of estimating them.El-Felaha J. 46(5):466-476. (In Arabic).

Satapathi, D.; R.C. Misra and B.S. Panda (1987) Variability, correlation and path coefficient analysis in linseed. J. Oil seed Res., 4(1):28-34.

Snedecor, G.M. and W.G. Cochran (1982).Statistical Methods.eight edition. Iowa State College, Press, Ames. Iowa, USA.

Svab.J. (1973).Biometric modszerek a lutusban Mezogazdassagi Kiado, Budapest. 


\title{
دراسات على متوسط السلوك و معاملى الارتباط و المرور لبعض التراكيب الوراثية من الكتان تحت ظروف بيئية مختلفة
}

\author{
جمال الدين حسين الثيمى ، مهذى محمد مهذى حسين و سناء سعيد حسن
}

أجرى هذا البحث بهذف تقييم ستة عشر تركيب وراثى من الكتان ممثلة فى أربعة عشر سلالة من الكتان و التى تمثل الطراز الثنائى (الألياف و

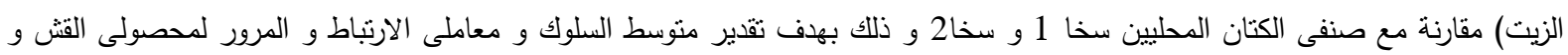
البذرة ومكوناتهما وذللك تحت ستة بيئات مختلفة (ثلاثة مواقع X موسمين) والثلاثة مواقع عبارة عن محطة بحوث كفر الحمام - محافظة الثرقية (أرض طينية) و التى تمثل الموقع الأول (L1) ، محطة بحوث الاسماعلية - محافظة الاسماعلية (أرض رملية) و النى تمثل الموقع الثانى (L2)

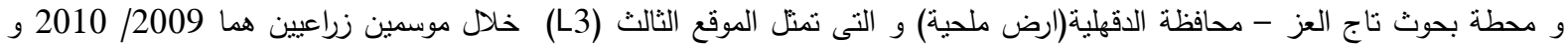

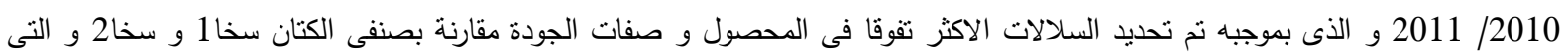

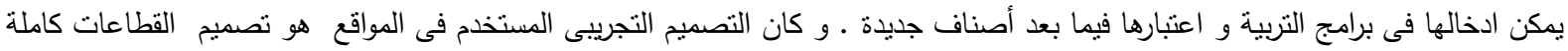
العشوائية ذات الثلاثة مكررات وقد اتبعت جميع العمليات الزراعية فى حدودها المنلى وطبقا لمنطقة الزراعة فى كل موفع من ميعاد زراعة و تسكيد

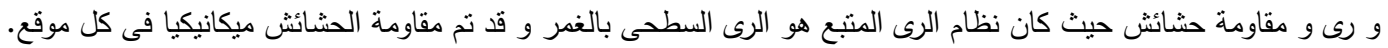

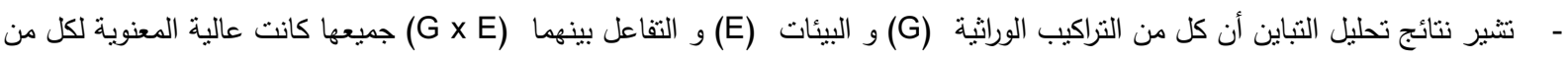
صفات القش و البذرة مما يدل على الاختلاف الواسع بين كل من التزاكيب الوراثية و البيئات و كذللك اختلاف استجابة هذه التزاكيب الوراثية التئية

للظروف البيئية. - أظهر تحليل التباين المشترك لكل من البيئات الثلاثة و موسمى الزراعة تفوق السلالة الثنائية 2/3/31 متبوعة بالسلالة 4/3/413 على باقى السلالات المختبرة و كذلك الصنفين المحليين سخا1 و سخا2 فى صفات محصول القش و المثنلة فى محصول القش للفان، محصول الالياف

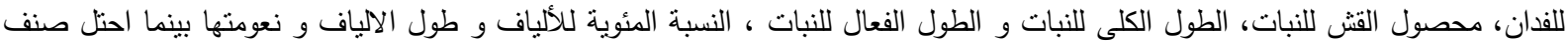

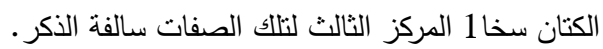
- سجلت السلالة الزيتية 16 و المتبوعة بالسلالة ال زيتية 2 اعلى القيم لصفات محصول البذرة و الممثلة فى محصول البذرة للفدان، محصول

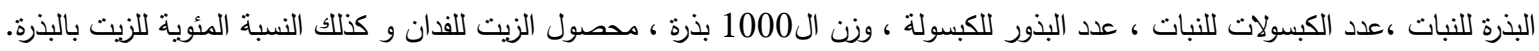

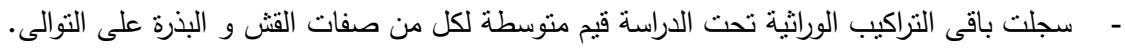

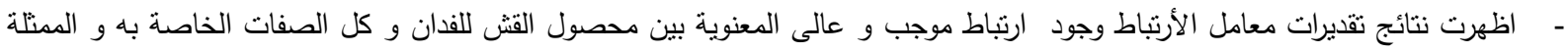
فى محصول الالياف للفدان ، محصول القش للنبات ، الطول الكلى و الطول الفعال للنبات ، النسبة المئوية للالياف و طول الالياف و نعومنها. من ناحية اخرى ارتبط محصول البذرة للفدان ارتباطا موجبا وعالى المعنوية بكل من محصول الزيت للفدان ، محصول البذرة للنبات ، عدد كبسولات

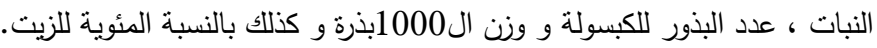

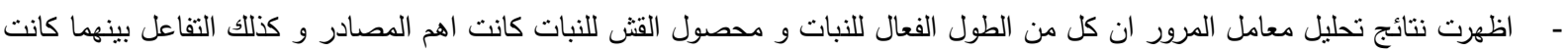
من اهم المصادر الرئيسية الدكونة لدحصول القش للفدان بمساهمة نسبية تقدر ب 39.21 ، 9.56 و 36.31\% على التوالى.فى نفس الوقت

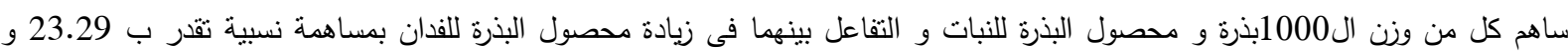
11.78 24.81 \% على النقالى.

- لتعظيم انتاجية و جودة محصولى القش و البذرة يجب على مربى النبات ان بعطى الهتماما كبيرا بان يضع فى اعتباره الانتخاب لصفة الطول

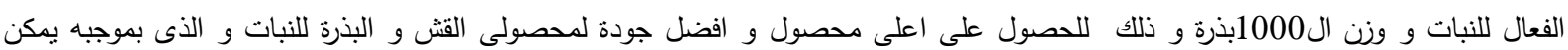
معظمة انتاجية و جودة محصول وحدة المساحة من الالياف و البذرة و الزيت على النقالى التوالى. - ـ تحت ظروف هذه الدراسة يمكن النوصية بالتوسع فى زراعة السلالة الثنائية 2/3/31 للحصول على اعلى محصول من القش كما و نوعا و

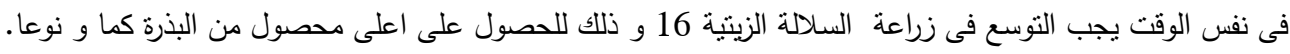

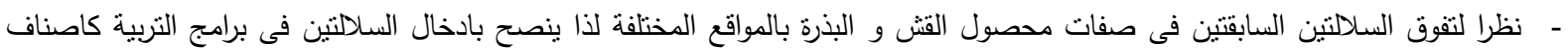

